\title{
Comportamiento de machos cebú en corrales presacrificio y su relación con el pH de la carne
}

\author{
Córdoba, C.P. ${ }^{1}$; Correa, G. ${ }^{2}$; Barahona, R. ${ }^{3}$ y Tarazona, A. ${ }^{3}$
}

'Fundación Hábitat, Territorio y Medio Ambiente. Medellín. Colombia.

2Departamento de Producción Animal. Universidad Nacional de Colombia. Facultad de Ciencias Agrarias. Medellín. Colombia. ${ }^{3}$ Departamento de Ingeniería Agronómica. Universidad Nacional de Colombia. Facultad de Ciencias Agrarias. Medellín. Colombia.

PALABRAS CLAVE ADICIONALES

Ayuno.

Presacrificio.

Temperatura.

Transporte.

\section{RESUMEN}

La adaptación de un animal al medioambiente está influenciada por varios factores, entre ellos los métodos utilizados para la reproducción, producción y sacrificio. El objetivo de este estudio fue evaluar el comportamiento individual de machos cebú en corrales presacrificio y relacionarlo con el pH de la carne. Un total de 150 novillos de la raza Brahman, entre 24 y 27 meses de edad y un peso promedio de $447,4 \pm 35,4 \mathrm{~kg}$, fueron estudiados en su etapa previa al sacrificio, evaluando los comportamientos agonistas de cada individuo durante seis horas posteriores al desembarque en la Central Ganadera de Medellín. El método utilizado para medir el comportamiento fue un registro continuo en el momento de ocurrencia de alguno de los comportamientos agonistas. Fueron estimadas las relaciones entre el tiempo de transporte (TT), el tiempo de ayuno (TA), el peso vivo (PV), la temperatura ambiental, humedad relativa, y la temperatura superficial (ventral (TV) y dorsal (TD)l, con el pH de la carne a las 24 horas post mortem y los comportamientos agonistas de los animales. Se concluye que un $\Pi$ superior a 20 horas desde la finca hasta la planta de sacrificio, y un TA mayor a 38 horas, implican un aumento en la actividad y la agresividad de los animales durante su espera en corrales. La TV y TD al momento de llegada al corral, influencian el descenso apropiado del $\mathrm{pH}$ de la carne. Los cambios en temperaturas superficiales a diferentes momentos, pueden aumentar el comportamiento agresivo, y además, PV superiores a $447 \mathrm{~kg}$ al momento del sacrificio, pueden afectar el descenso adecuado del $\mathrm{pH}$.

\section{Zebu males behavior in pre slaughter pens and relationship to meat $\mathrm{pH}$}

\section{SUMMARY}

\section{ADDITIONAL KEYWORDS}

Fasting.

Preslaughter.

Temperature.

Transport.

\section{INFORMATION}

Cronología del artículo.

Recibido/Received: 20.06 .2016

Aceptado/Accepted: 20.09.2017

On-line: 15.10 .2017

Correspondencia a los autores/Contact e-mail:

ccordoba@grupohtm.org

\section{INTRODUCCIÓN}

El bienestar y el comportamiento animal han sido objeto de estudio en múltiples investigaciones durante las últimas décadas. Cada vez existe más evidencia científica que demuestra que animales sometidos a situaciones estresantes reaccionan negativamente tanto en su relación con otros individuos, como en su rendimiento y calidad del producto final (Romero et al., 2013). Prácticas inadecuadas de marcación, vacunación, castración, pesaje, transporte y sacrificio, producen en los bovinos deshidratación, miedo y au- mento de la actividad física; ocasionando estrés, fatiga y lesiones que afectan su bienestar (Fergusony Warner, 2008; Romero et al., 2013; Muñoz, 2014). Dependiendo de la magnitud, duración e intensidad de los agentes estresores y de la susceptibilidad de los animales, su temperamento y bienestar se pueden afectar de manera directa (Ferguson y Warner, 2008). Por lo tanto, en el estudio del bienestar animal se necesita seguir un enfoque interdisciplinario que reúna a investigadores de diferentes disciplinas y que complemente los enfoques tradicionales de las ciencias naturales; de este modo 
mejorará la comprensión del bienestar animal, a la vez que se obtienen ganancias metodológicas para realizar futuras investigaciones sobre el manejo racional del ganado (Carenzi y Verga, 2009). La etapa presacrificio es una de las más estresantes para los bovinos, la logística que esta etapa implica ocasiona estrés y pérdidas económicas al disminuir la calidad del producto (Gallo, 2008; Miranda, 2013); por ello se hace necesario entender mejor el comportamiento animal presacrificio, especialmente la interacción entre amenazas, luchas y huidas (comportamientos agonistas). El control de comportamientos agonistas es necesario durante las rutinas de manejo, tanto entre los animales como hacia el ser humano (Petryna y Bavera, 2002). Si la agresividad, el estrés y los ataques son recurrentes, el manejo de un animal o un grupo será afectado, haciéndolo complicado y peligroso para el encargado (Miranda, 2013). Se ha reportado que el tiempo de transporte prolongado aumenta las pérdidas de peso vivo entre 1,5 y $9 \%$ e incrementa riesgos de caídas, muertes y contusiones; lo cual conlleva pérdidas económicas por eliminación de tejido contuso, menor rendimiento en canal y descenso en la categoría de tipificación de las canales (Gallo, 2008; Romero et al., 2013). Con 3 a 12 horas de viaje, las pérdidas de peso vivo en novillos fluctúan entre 5 y $9 \%$, y viajes de 24 horas pueden alcanzar un $12 \%$ de pérdida (Gallo et al., 2000; 2001). Respecto a las contusiones, si el transporte es menor a 8 horas, se han registrado $7,92 \%$ de canales con contusión, mientras que con transportes mayores a 24 horas las contusiones en la canal han ascendido a 10,48\% (Herrera, 2008). Conociendo entonces que la etapa presacrificio influye directamente en el rendimiento y calidad de la carne, se hace necesario ampliar el conocimiento y relaciones del comportamiento animal con todo lo que el presacrificio conlleva.

Como aporte a esta construcción de conocimiento, el presente estudio tuvo como objetivo la caracterización del comportamiento individual de novillos cebú durante la espera en corrales presacrificio, relacionando dicho comportamiento con el $\mathrm{pH}$ de la carne 24 horas post mortem y con otras variables que pueden influir en la alteración del comportamiento bovino presacrificio.

\section{MATERIALES Y MÉTODOS}

\section{LOCALIZACIÓN}

La investigación se llevó a cabo en las instalaciones de la Central Ganadera S.A., en donde se realiza comercialización y sacrificio de ganado proveniente de diversas zonas del país. Está ubicada en la ciudad de Medellín, Colombia a 1465 m.s.n.m., con una precipitación promedia anual de $1500 \mathrm{~mm}$, humedad relativa del $76 \%$, temperatura promedio de $21^{\circ} \mathrm{C}$ y clasificada de acuerdo a Holdridge (1967) en la formación ecológica de Bosque húmedo premontano (Bh-p).

\section{ANIMALES EXPERIMENTALES Y ALIMENTACIÓN}

Los animales evaluados pertenecían a la empresa comercial AP3 Carnes y provenían de predios ganaderos en los departamentos de Sucre y Córdoba, ubicados al norte de Colombia. La distancia de estos departamentos hasta la Central Ganadera, es de 552 y 395 kilómetros respectivamente.

En el experimento se evaluaron 150 novillos entre 24 y 27 meses de edad, de genética similar (Brahman comercial). Su sistema de alimentación era pastoreo rotacional con potreros de 0,5 a 1 hectárea, con sal a voluntad y bebederos para el suministro de agua. El peso promedio al sacrificio de dichos animales fue de $447,4 \pm 35,4 \mathrm{~kg}$. Durante su estadía en la planta de beneficio los animales tuvieron acceso a agua a voluntad. Durante el transporte no hubo oferta de alimento ni agua.

\section{REGISTRO DE VARIABLES PRESACRIFICIO Y EVALUACIÓN DEL COMPORTAMIENTO}

Al momento de desembarcar cada animal fue pesado y el tiempo de transporte reportado desde la salida de la finca hasta el momento de llegada a la Central Ganadera. El tiempo de ayuno se consideró desde el reposo en finca hasta el sacrificio, así: tiempo entre la última vez que el animal consumió alimento sólido y el comienzo del transporte, tiempo de transporte y tiempo de permanencia en los corrales de espera presacrificio. Durante el pesaje, cada animal fue enumerado consecutivamente para facilitar su identificación en los corrales. Los números fueron hechos en tres puntos anatómicos (posterior a la última costilla y en la parte trasera en el anca), a ambos lados del animal. Para ello no se utilizaron tintes o tatuajes, sino la pintura azul de marcación que se emplea normalmente en la Central Ganadera. Posterior a la marcación, se tomó la primera temperatura superficial y se verificó la procedencia de cada animal. Ya en los corrales, la temperatura superficial fue medida cada 3 horas, utilizando un termómetro Infrarrojo Fluke 561, que permite hacer la medición hasta a un metro de distancia sin necesidad de perturbar o modificar la actividad que el animal este realizando (Tarazona, 2013; Ceballos et al., 2011). Para evitar factores de variación al tomar la temperatura, esta se hizo siempre en la piel limpia, a la misma distancia y en los mismos puntos para todos los animales.

El comportamiento se observó durante las 6 primeras horas de espera en corrales presacrificio, en 11 grupos de animales, para un total de 150 bovinos de carne. El tiempo de observación fue establecido teniendo en cuenta la ley en Colombia para el reposo de bovinos en plantas de beneficio (Decreto 1500 de 2007 y Resolución 2905 de 2007). Además, por observaciones previas se determinó que después de 6 horas de espera en corral, los animales disminuían su actividad y su patrón de comportamiento era descansar echados. La observación fue hecha en verano, entre los meses de octubre y febrero, con un promedio de temperatura ambiental de $23,79 \pm 0,74^{\circ} \mathrm{C}$, y una humedad relativa promedio de $59,09 \pm 6,58$.

El tiempo de observación inició inmediatamente después del desembarque y pesaje. Dos personas previamente entrenadas estuvieron a cargo de observar cada grupo. El entrenamiento tuvo como referencia las observaciones de un investigador experimentado hasta lograr confiabilidad entre ellos. El método utilizado fue un registro continuo durante las 6 horas de evaluación, donde se registraban los comportamientos agonistas y 
las respuestas de cada animal, en el mismo momento en que ocurría el evento (Martín y Bateson, 2007). Los eventos se definieron como: $a$. comportamientos agresivos del emisor: empujar (cuando un animal usando la cabeza hace presión constante sobre otro animal hasta desplazarlo), cabecear (cuando un animal toca con fuerza a otro usando la cabeza) y perseguir (cuando un animal se dirige hacia a otro, lo desplaza y continúa tras él); $b$. comportamientos de respuesta pasiva: indiferencia (cuando un animal no responde a la agresión), huida (cuando el animal objeto de la agresión se desplaza de su lugar); c. comportamientos agresivos del receptor (respuesta): empujar o cabecear.

No fue posible realizar habituación de los animales a la presencia de los observadores, debido a que el estudio se realizó en las condiciones normales y comerciales de los corrales de espera, donde hay presencia y flujo de personas continuamente. Los observadores se ubicaron en la periferia del corral, en diferentes puntos para evitar la pérdida de registros.

Finalmente, fueron estimadas y analizadas las relaciones entre tiempo de transporte (TT), tiempo de (TA), peso vivo (PV), temperatura superficial del animal (ventral (TV) y dorsal (TD)), temperatura ambiental y humedad relativa, con el $\mathrm{pH}$ de la carne a las 24 horas post mortem y el comportamiento agonista de los animales.

\section{SACRIFICIO DE LOS ANIMALES Y MEDICIÓN DEL PH A LAS 24 HORAS}

El sacrificio de los animales fue realizado en la Planta de beneficio de la Central Ganadera, de acuerdo con el Decreto 1500 de 2007 y Resolución 2905 de 2007 de la ley Colombiana. El pH se evaluó a las 24 horas de refrigeración de las canales, midiéndolo en el músculo Longissimus dorsi con un potenciómetro portátil Schott.

\section{ANÁLISIS ESTADÍSTICO}

Se ajustaron modelos de regresión múltiples y simples para el número de eventos agonistas (variable dependiente) en función del tiempo de transporte, tiempo de ayuno, peso vivo, temperaturas superficiales del animal, temperatura ambiental y humedad relativa (variables independientes). Asimismo, se ajustaron modelos de regresión para evaluar el efecto del número de comportamientos agonistas sobre el pH de la carne a las 24 horas post mortem y de los factores relacionados con transporte y espera en corrales sobre esta variable. El programa usado fue Statgraphics Centurión XVI versión 16.1.02.

\section{RESULTADOS Y DISCUSIÓN}

\section{TIEMPO DE TRANSPORTE}

Se encontró una alta relación $\left(\mathrm{R}^{2} 0,9922\right)$ entre el tiempo de transporte y la presentación de comportamientos agresivos. El transporte promedio fue de $23,1 \pm 2,57$ horas desde el embarque en finca hasta el descargue en la central ganadera, y las acciones agresivas (del emisor y el receptor) variaron entre 23 y 270 eventos, durante las 6 horas de observación, según el grupo. Se determinó que por cada hora adicional en tiempo de transporte, se incrementa en un 4,5\% el nú- mero de eventos agonistas en el corral; tanto agresiones como respuestas agresivas (figura 1). Este resultado es acorde con otros estudios, donde se expresa que durante el manejo presacrificio el ganado se enfrenta a problemas graves de bienestar (Warriss et al., 1995; Knowles, 1999; Gallo, 2009). Mientras sucede el embarque, transporte, desembarque y espera en corrales, el animal tiene que encontrar estrategias para hacer frente a una variedad de factores físicos, climáticos, psicológicos o sociales, que son estresantes (Ferguson y Warner, 2008; Adzitey, 2011).

Un factor estresante para los bovinos que puede inducir agresión, es el miedo causado por eventos desconocidos como ruidos, sombras o movimientos bruscos (Dantzer y Mormede, 1983; Grandin et al., 1998). Además, por su gran extensión, sus características geográficas peculiares y por la existencia de rutas no pavimentadas de difícil acceso, el transporte de ganado se prolonga por más tiempo del esperado independientemente de los kilómetros recorridos (Gallo, 2008; Gallo y Tadich, 2008). Incluso en buenas condiciones de transporte los animales muestran signos de estrés y lesiones en diferentes grados. Se ha reportado que el transporte por cualquier medio, aunque sea corto, puede afectar las condiciones físicas del animal, así como la calidad de la carne y su vida útil, al modificar las condiciones de acidez muscular, el tiempo de aparición del rigor mortis y su duración; incluso la ubicación que los animales lleven en el camión (compartimiento trasero o delantero) influencia la frecuencia cardiaca o algunos indicadores sanguíneos como el cortisol (Van de Water et al., 2003; Paranhos da Costa y Tarazona, 2011).

Lo anterior sugiere que el estrés durante el transporte, más aún si este es prolongado, provoca cambios en el comportamiento de los bovinos, haciéndolos más agresivos con sus compañeros de corral y más reactivos ante cualquier situación externa, además, dichas alteraciones de comportamiento y estrés presacrificio podrían influir también en la calidad final de la carne.

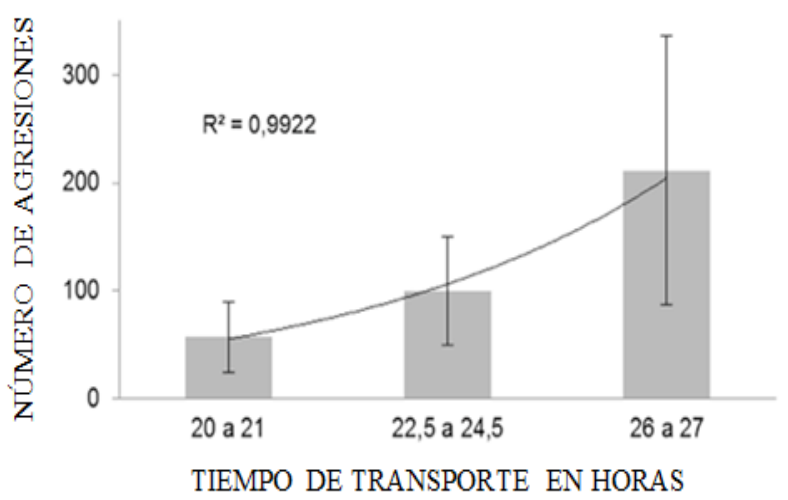

Figura 1. Relación entre los comportamientos agresivos de machos cebú durante las primeras 6 horas en corral de espera y el tiempo de transporte presacrificio (Relationship between the aggressive behaviors of zebu males during the first 6 hours in the holding pen and the pre-slaughter transport time). 
Tabla I. Distribución poblacional de los machos cebú de acuerdo al valor de $\mathrm{pH}$ del lomo Longissimus dorsi 24 horas post mortem (Population distribution of zebu males accordingto $\mathrm{pH}$ value of Longissimus dorsi 24 hours post mortem).

\begin{tabular}{lcc}
\hline $\begin{array}{l}\text { pH 24 horas post } \\
\text { mortem }\end{array}$ & $\begin{array}{c}\text { Número de } \\
\text { animales }\end{array}$ & $\begin{array}{c}\text { Porcentaje de la } \\
\text { población }\end{array}$ \\
\hline Menor a 5,4 & 2 & $1,3 \%$ \\
Entre 5,4 y 5,8 & 12 & $8 \%$ \\
Entre 5,8 y 6 & 34 & $22,7 \%$ \\
Superior a 6 & 102 & $68 \%$ \\
\hline
\end{tabular}

\section{PH DEL LOMO (LONGISSIMUS DORSI) A LAS 24 HORAS}

El promedio de $\mathrm{pH}$ para los 150 lomos evaluados, fue de $6,25 \pm 0,42$. El $68 \%$ de los animales evaluados produjo carnes con valores de $\mathrm{pH}$ superiores a 6,0 (tabla I), lo cual sugiere estrés previo al sacrificio, consumo excesivo de glucógeno muscular y disminución en la formación de ácido láctico en el músculo post mortem (Soria y Corva, 2004; Gallo y Tadich, 2008).

Para bovinos el $\mathrm{pH}$ ideal de la carne a las 24 horas post mortem está entre 5,4 a 5,8 (Warriss, 2000; Gallo y Tadich, 2008; Miranda, 2013). Un pH superior sugiere una carne más oscura, dura y seca, anomalía que en el bovino se conoce como corte oscuro (Hood y Tarrant, 1981) o carne DFD. Las carnes DFD son un indicador de pobre bienestar, ya que evidencian el consumo acelerado del glucógeno muscular, relacionado con estrés, fatiga, ayuno, lesiones o enfermedad (Costa, 2013; Quiroz et al., 2016).

\section{VARIABLES AMBIENTALES}

Es sabido la importancia de las condiciones medioambientales para la termorregulación y confort animal (Soria y Corva, 2004; Espinoza et al., 2011; Tarazona, 2013; Benavides et al., 2016), aun así, en este estudio la temperatura ambiental y la humedad relativa no tuvieron diferencia significativa $(p>0,05)$ respecto al pH del lomo a las 24 horas o a la presentación de eventos agresivos en el corral de espera presacrificio. Aunque la mayoría de los grupos fueron observados en días diferentes, todo el estudio se hizo en verano y las variaciones climáticas entre días no fueron estadísticamente significativas, por ello, la variación de temperatura y humedad no influyó ni el comportamiento agresivo ni en el $\mathrm{pH}$ a las 24 horas de los animales en este estudio. En la tabla II, se presentan los diferentes grupos evaluados, el número de animales en cada uno de ellos y los valores de temperatura ambiental y humedad relativa para cada día de muestreo. Dicha tabla se complementa además, con las horas de ayuno que tuvo cada grupo evaluado.

\section{AYUNO}

En la tabla III se muestra el promedio y desviación estándar para las variables ayuno y temperatura superficial (ventral y dorsal) de los animales en este estudio. El tiempo de ayuno de los grupos evaluados fluctuó entre 38 y 45 horas, con un promedio de de $41,4 \pm 1,9$ horas. El tiempo de ayuno en este estudio tuvo relación estadísticamente significativa $(\mathrm{p}<0,05)$ con el $\mathrm{pH}$ del lomo, explicando un 16\% de la variación del $\mathrm{pH}$. $\mathrm{Al}$ aumentar el tiempo de ayuno, aumenta el pH de la carne (figura 2). Esta relación puede explicarse ya que la estructura de las miofibras, el contenido de glucógeno y la actividad proteolítica interactúan con el estrés presacrificio, determinando así algunas características finales de la calidad de la carne (Oddy et al., 2001).

El ayuno en todos los grupos evaluados fue superior a 38 horas, y en gran medida esto se debe al largo tiempo de transporte (promedio de $23,1 \pm 2,5 \mathrm{~h}$ ) y de espera en corrales (promedio de 14,4 $\pm 1,6 \mathrm{~h}$ ), afectando finalmente el $\mathrm{pH}$ de la carne. Se ha reportado que ayunos mayores o iguales a 24 horas aumentan la probabilidad de presentar canales con $\mathrm{pH} \geq 5,8$ (Amtmann et al., 2006); además, que el ayuno por sí solo, no causa apreciables pérdidas de glucógeno muscular, sino cuando es acompañado de factores estresantes como la restric-

Tabla II. Número de animales por grupo de evaluación en corrales de espera presacrificio, temperatura ambiental, humedad relativa y ayuno total en los diferentes días de evaluación (Number of animals per evaluation group in pre-slaughter holding pens, ambient temperature, relative humidity and total fasting on the different evaluation days).

\begin{tabular}{|c|c|c|c|c|c|c|}
\hline \multirow[t]{2}{*}{ Grupo } & \multirow{2}{*}{$\begin{array}{l}\text { Número de } \\
\text { animales }\end{array}$} & \multicolumn{3}{|c|}{ Temperatura ambiental $\left({ }^{\circ} \mathrm{C}\right)$} & \multirow{2}{*}{$\begin{array}{c}\text { Humedad relativa } \\
(\%)\end{array}$} & \multirow{2}{*}{$\begin{array}{l}\text { Ayuno total } \\
\text { (horas) }\end{array}$} \\
\hline & & Promedio & Min & Max & & \\
\hline 1 & 14 & 22,9 & 18 & 28 & 64 & 38 \\
\hline 2 & 13 & 24,1 & 19 & 29 & 63 & 42 \\
\hline 3 & 13 & 24,1 & 19 & 29 & 63 & 40 \\
\hline 4 & 13 & 22,7 & 18,6 & 26 & 68 & 41 \\
\hline 5 & 14 & 22,7 & 18,6 & 26 & 68 & 41,5 \\
\hline 6 & 14 & 23,9 & 17 & 28 & 54 & 42 \\
\hline 7 & 13 & 23,9 & 17 & 28 & 54 & 45 \\
\hline 8 & 14 & 24,8 & 19,2 & 29 & 50 & 42 \\
\hline 9 & 14 & 24,8 & 19,2 & 29 & 50 & 43 \\
\hline 10 & 14 & 23,9 & 18 & 28 & 58 & 39 \\
\hline 11 & 14 & 23,9 & 18 & 28 & 58 & 42 \\
\hline
\end{tabular}


Tabla III. Promedio y desviación estándar para el ayuno presacrificio y temperaturas superficiales de machos cebú en corrales de espera (Average and standard deviation for pre-fasting and surface temperatures of zebu males in waiting pens).

\begin{tabular}{lcc}
\hline Variable & Promedio & DE \\
\hline Ayuno & 41,4 horas & $\pm 1,91$ \\
TSV a la llegada & $32,5^{\circ} \mathrm{C}$ & $\pm 1,79$ \\
TSV a las 3 horas & $33,4^{\circ} \mathrm{C}$ & $\pm 2,00$ \\
TSV a las 6 horas & $33,5^{\circ} \mathrm{C}$ & $\pm 1,79$ \\
TSD a la llegada & $31,2^{\circ} \mathrm{C}$ & $\pm 2,06$ \\
TSD a las 3 horas & $31,0^{\circ} \mathrm{C}$ & $\pm 1,50$ \\
TSD a las 6 horas & $31,8^{\circ} \mathrm{C}$ & $\pm 1,70$
\end{tabular}

TSV: temperatura superficial del vientre. TSD: temperatura superficial del dorso. DE: Desviación estándar.

ción de libertad, el manejo, el enfrentar lo desconocido, sed, fatiga, injurias y temperaturas extremas (Ashmore et al., 1973; Grandin, 1997). El efecto que tiene la carencia de alimento en bovinos difiere según su duración (horas); los tiempos de ayuno prolongados (más de 24 horas) pueden provocar pérdidas de peso, disminución del rendimiento en canal, así como alteraciones de la calidad de la carne, entre ellos el descenso del pH en la canal (Gallo y Tadich, 2008; Mota et al., 2016; Quiroz et al., 2016), siendo esto último corroborado en el presente estudio.

\section{PESO VIVO PRESACRIFICIO}

El PV promedio al llegar al corral, fue de 447,3 \pm $23,9 \mathrm{~kg}$. Existió una relación significativa $(\mathrm{p}=0,0359)$ entre PV y $\mathrm{pH}$. A mayor PV presacrificio, el $\mathrm{pH}$ de la carne es $16 \%$ mayor. Esto corrobora que los animales más pesados tienden a movilizar una mayor proporción de sus tejidos para proporcionar la energía necesaria y mantener las funciones vitales durante las estresantes condiciones presacrificio (Warriss, 1992; Alarcón y Janacua, 2012).

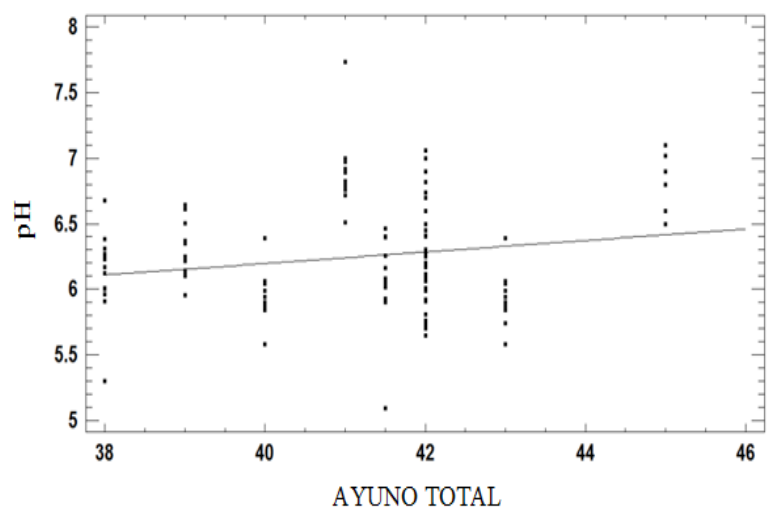

Figura 2. Relación del ayuno total previo al sacrificio de machos cebú, con el pH de su lomo a las 24 horas post mortem (Relationship of total fasting prior to slaughter of zebu males, with loin $\mathrm{pH}$ at 24 hours post mortem).
Tabla IV. Valor p para el modelo de regresión lineal simple entre variables predictoras (ayuno y temperatura superficial) y variables respuesta (agresiones y pH de la carne) de machos cebú presacrificio ( $p$ value for the simple linear regression model between predictor variables (fasting and surface temperature) and response variables (aggressions and $\mathrm{pH}$ of the meat) of zebu males pre-slaughter).

\begin{tabular}{lcc}
\hline \multirow{2}{*}{$\begin{array}{l}\text { Variables } \\
\text { predictoras }\end{array}$} & \multicolumn{2}{c}{ Variables respuesta } \\
\cline { 2 - 3 } Ayuno & 0,1478 & $0,0210^{*}$ \\
TSV a la llegada & 0,6086 & $0,0021^{*}$ \\
TSV a las 3 horas & 0,2309 & 0,6598 \\
TSV a las 6 horas & $0,0085^{*}$ & $0,0424^{*}$ \\
TSD a la llegada & 0,6859 & $0,0000^{*}$ \\
TSD a las 3 horas & $0,0014^{*}$ & 0,0803 \\
TSD a las 6 horas & $0,0008^{*}$ & 0,6817 \\
\hline *Relación significativa &
\end{tabular}

\section{TEMPERATURA SUPERFICIAL}

La temperatura superficial en este estudio influenció tanto la ocurrencia de eventos agresivos como el pH a las 24 horas postmortem. $\mathrm{Al}$ aumentar en $1^{\circ} \mathrm{C}$ la TSV a las 6 horas, la TSD a las 3 y la TSD a las 6 horas de espera en corral presacrificio, el número de agresiones por animal aumenta en 1,18; 1,26 y 1,14 eventos; respectivamente. Dichos eventos hacen alusión no sólo a las agresiones por parte de un emisor, sino también a las respuestas agresivas que tiene el receptor de dichas agresiones.

Este hallazgo puede explicarse con las respuestas fisiológicas generadas por el estrés calórico; ya que los mamíferos en su intento de termoregularse liberan catecolaminas como noradrenalina y adrenalina, encargadas de poner al animal en estado de alerta, influenciando comportamientos de lucha y huida (Romero y Sanchéz, 2011).

Las variables que se relacionaron significativamente $(\mathrm{p}<0,05)$ con el $\mathrm{pH}$ de la carne, fueron: TSV a la llegada a corrales, TSV a las 6 horas de espera y TSD a la llegada al corral. En la tabla IV, se muestra el valor p para cada una de las variables mencionadas.

$\mathrm{Al}$ aumentar en $1^{\circ} \mathrm{C}$ la TSD a la llegada a corral, el $\mathrm{pH}$ a las 24 horas aumentó en 0,09 (figura 3). La TSV, al igual que la TSD, puede aumentar el pH del lomo a las 24 horas. Para este estudio, un 7\% de la variación del $\mathrm{pH}$, es explicado por cambios en la temperatura ventral. Tal como se muestra en la figura 4, hubo diferencia entre la TSV al desembarque y la TSV a las 6 horas de espera en corral.

Se observó que el diferencial de temperatura ventral (temperatura a las 6 horas menos la temperatura de desembarque) estuvo lineal y positivamente relacionado con las temperaturas de llegada de los animales (figura 5). Esto sugiere que algunos animales por medio de mecanismos de termorregulación, aumentaron o disminuyeron su temperatura después de 6 horas de haber llegado al corral, afectando así el descenso esperado del $\mathrm{pH}$ a las 24 horas post mortem. 


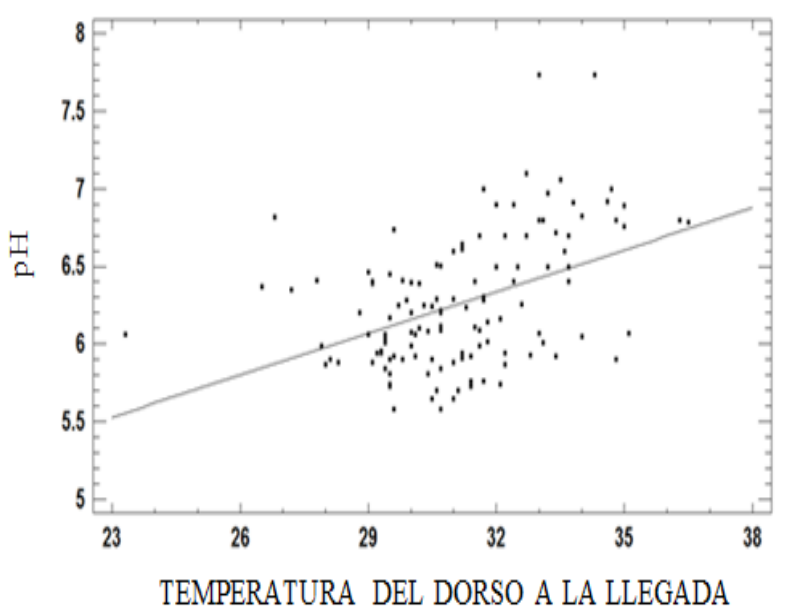

Figura 3. Relación de la temperatura $\left({ }^{\circ} \mathrm{C}\right)$ dorsal de machos cebú a la llegada al corral de espera presacrificio, con el $\mathrm{pH}$ del lomo 24 horas post mortem (Relationship of the dorsal temperatura $\left({ }^{\circ} \mathrm{C}\right)$ of zebu males to the arrival at the corral of presacrificial wait, with the $\mathrm{pH}$ of the loin 24 hours post mortem).
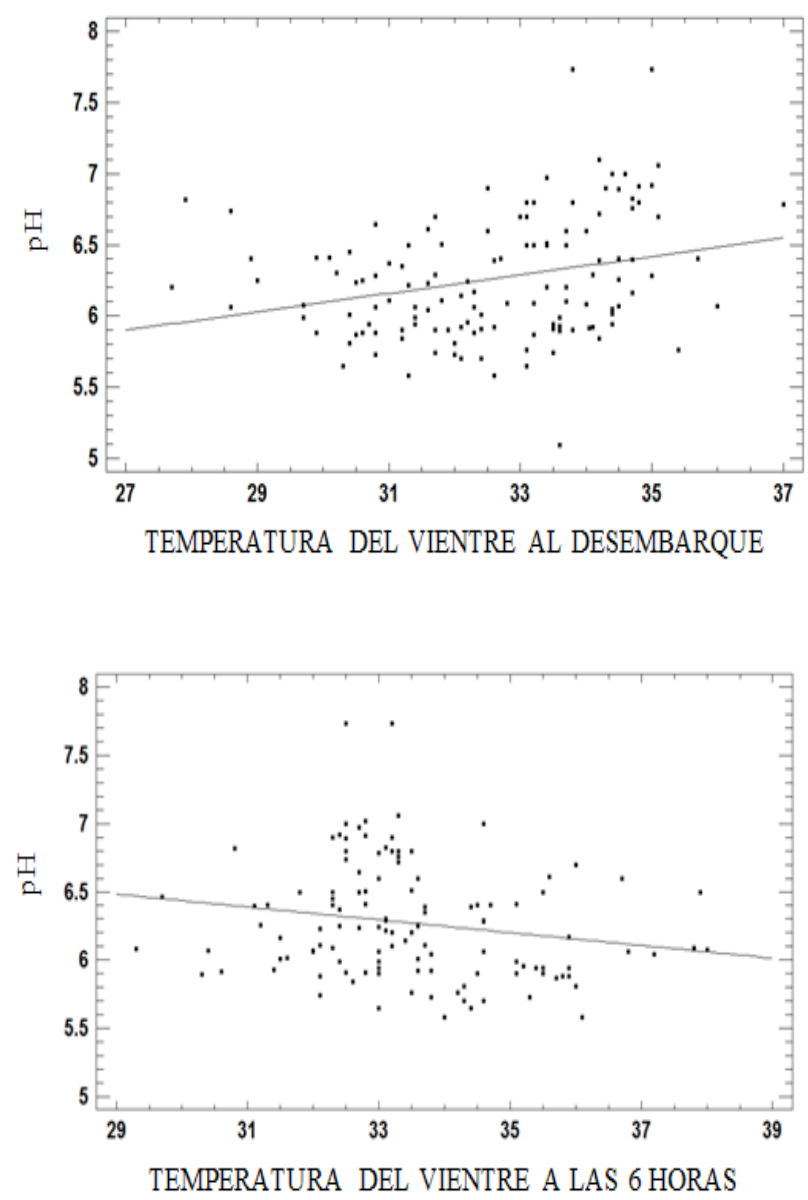

Figura 4. Relación de la temperatura superficial $\left({ }^{\circ} \mathrm{C}\right)$ del vientre al desembarquede machos cebú y la temperatura a las 6 horas de espera en corral presacrificio, con el pH del lomo a las 24 horas post mortem (Relation of the ventral superficial temperature to the landing of zebu males and the temperature at 6 hours of waiting in corral presacrificio, with the $\mathrm{pH}$ of the spine at 24 hours post mortem).

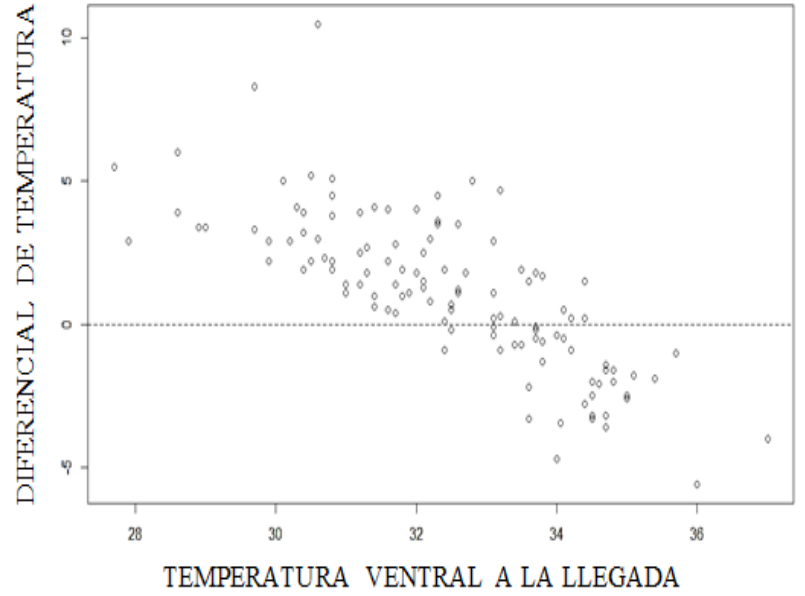

Figura 5. Diferencial de temperatura $\left({ }^{\circ} \mathrm{C}\right)$ ventral de machos cebú a la llegada y a las 6 horas de espera en corral presacrificio (Ventral temperatura differentia $I\left({ }^{\circ} \mathrm{C}\right)$ of zebu males on arrival and at 6 hours of waiting in corral presacrificio).

Mientras más calientes llegan los animales a la Central Ganadera, más tienden a perder calor durante las 6 horas siguientes y viceversa. Se estima que la temperatura neutral estuvo entre los 33 y $35^{\circ} \mathrm{C}$, pues animales que llegaron con dicha temperatura tendieron a permanecer igual a las 6 horas de espera en corral. La relación hallada entre la variación de temperatura superficial y el $\mathrm{pH}$, podría explicarse desde dos puntos de vista: $a$. La necesidad de termorregulación de los animales: Al necesitar glucógeno para los procesos de termorregulación, se generan pérdidas de las reservas corporales y por lo tanto reducción de la productividad animal (Warriss, 2000). b. La fisiología muscular del animal durante el transporte, la espera y el ayuno: La intensa actividad física de los animales hacinados en un camión para su transporte, sin sitios de apoyo y a través de carreteras difíciles, causa el agotamiento de las reservas de glucógeno muscular (Lenis et al., 2016; Sanmiguel y Díaz, 2011). El efecto hiperglicemiante del cortisol y las catecolaminas adrenales inducen un estado catabólico en el animal con la consecuente movilización de las reservas energéticas que no pueden reponerse debido a la inanición por el ayuno (Warriss, 1992). Sin reservas de glucógeno disminuirá drásticamente la contracción muscular refleja post mortem, manifestándose en una menor disminución del pH en las canales (Warriss, 2000; Soria y Corva, 2004).

Para este estudio se cree que las variaciones de TSV mencionadas entre animales (diferencial entre TSV a las 6 horas y al desembarque), dependen de su posición/ubicación en el camión durante el transporte. Los animales, aunque podían cambiar de posición dentro del camión, no podían desplazarse dentro de él; por lo cual algunos individuos siempre estuvieron expuestos a las corrientes de aire y otras condiciones ambientales directas (periferia del camión) que no se presentan en el centro del camión; por ende, los animales que llegan con menos temperatura ventral, tienden a ganar temperatura durante su instancia en corral, mientras que 
los animales que se presume fueron transportados en el centro del camión, llegan con temperatura ventral mayor y tienen a disminuirla en los corrales. Se sugiere ampliar investigación sobre este tema y tratar de mantener a los animales en su zona termoneutral durante el transporte y demás actividades presacrificio, por ejemplo: transportar a los animales al finalizar la tarde y en la noche, con el fin de evitar las mayores temperaturas del día; además, adecuar las instalaciones de los corrales de espera, ya que pisos de cemento, exposiciones directas al sol y ventilación reducida, pueden dificultar la termorregulación.

\section{CONCLUSIONES}

El aumento en el tiempo de transporte está asociado con aumentos en la presentación de comportamientos agonistas en los bovinos durante su espera en corrales presacrificio. Sin embargo, los aumentos en el tiempo de transporte no tuvieron relación con el pH a las 24 horas post mortem. La temperatura superficial, ventral y dorsal, también tuvieron relación con el número de comportamientos agresivos; a mayor temperatura, mayor cantidad de agresiones y respuestas agresivas.

La variación de temperatura superficial ventral a la llegada de los animales y a las 6 horas de espera en corral, así como el tiempo de ayuno y la temperatura dorsal a la llegada, tuvieron relación directa con el pH de la canal.

Se sugiere realizar más estudios en esta área, teniendo en cuenta otras variables como las medioambientales, de manipulación de los animales y su procedencia, con el fin de tener una mejor línea base que permita el diseño de protocolos presacrificio ajustados a las condiciones propias del sector cárnico.

\section{NOTA DE LOS AUTORES}

Se agradece a la empresa comercial AP3 Carnes y a la Central Ganadera de Medellín, por permitir el acceso a sus animales e instalaciones. Al proyecto Efecto del tiempo de transporte y espera ante-mortem sobre algunos biomarcadores de estrés bovino y la calidad sensorial de la carne financiado por Min Agricultura, evaluado por CENIRED y ejecutado por la UN sede Medellín, por facilitar el recurso humano necesario para los muestreos.

\section{BIBLIOGRAFÍA}

Adzitey, F. 2011. Effect of pre-slaughter animal handling on carcass and meat quality. Int Food Res J, 18: 485-491.

Alarcón, A.D. y Janacua, H. 2012. Alteración de las reacciones enzimáticas post-mortem en carnes pálidas, suaves y exudativas u oscuras, firmes y secas. En: Bienestar Animal - Productividad y calidad de carne. Editorial Elsevier. México. 499 pp.

Amtmann, V.; Gallo, C.; Van Schaik, G. y Tadich, N. 2006. Relaciones entre el manejo ante-mortem, variables sanguíneas indicado-ras de estrés y pH de la canal en novillos. Arch Med Vet, 38: 259-264.

Ashmore, C.; Carroll, F.; Doerr, L.; Tompkins, G.; Stokes, H. and Parker, W. 1973. Experimental prevention of dark - cutting meat. J Anim Sci, 36: 33-36.
Benavides, R.; Silva, F.; Perilla, S. y Sánchez, H. 2016. Caracterización del ambiente térmico para la actividad ganadera bovina en el Valle del Cauca, Colombia. Acta Agron, 65: 406-412.

Carenzi, C. and Verga, M. 2009. Animal welfare: review of the scientific concept and definition. Ital J Anim Sci, 8: 21-30.

Ceballos, M.; Cuartas, C.; Naranjo, J.; Rivera, J.; Arenas, F.; Murgueitio, E. y Tarazona, A. 2011 . Efecto de la temperatura y la humedad ambiental sobre el comportamiento de consumo en sistemas silvopastoriles intensivos y posibles implicaciones en el conforttérmico. RCCP, 24: 368 .

Costa, F. 2013. Efeitos do tempo de espera em currais de frigorífico no bem-estar e na qualidade da carne de bovinos. Dissertação (mestrado). Universidade Estadual Paulista. Faculdade de Ciências Agrárias e Veterinárias. Jaboticabal. Brasil.

Dantzer, R. and Mormede, P. 1983. Stress in farm animals: a need for re-evaluation. J Anim Sci, 57: 6-18.

Espinoza, J.; Ortega, P.; Palacios, A. y Guillén, A. 2011 . Tolerancia al calor y humedad atmosférica de diferentes grupos raciales de ganado bovino. Rev MVZ Córdoba, 16: 2302-2309.

Ferguson, D.M. and Warner R.D. 2008. Have we underestimated the impact of pre-slaughter on meat quality in ruminants? Meat Sci, 80: 12-19.

Gallo, C.; Pérez, S.; Sanhueza, C. y Basic, J. 2000. Efectos del tiempo de transporte de novillos previo al faenamiento sobre el comportamiento, las pérdidas de peso y algunas características de la canal. Arch Med Vet, 32: 157-170.

Gallo, C.; Espinoza, M. y Basic, J. 2001. Efectos del transporte por camión durante 36 horas, con y sin período de descanso sobre el peso vivo y algunos aspectos de calidad de carne bovina. Arch Med Vet, 33: 43-53.

Gallo, C. 2008. Using scientific evidence to inform public policy on the long distance transportation of animals in South America. Vet Ital, 44: 113-120.

Gallo, C. y Tadich, T. 2008. Bienestar animal y calidad de carne durante los manejos previos al faenamiento en bovinos. REDVET, IX: 1OB.

Gallo, C. 2009. Transporte y reposo pre-sacrificio en bovinos y su relación con la calidad de la carne. En: Bienestar Animal y Calidad de la Carne. Editorial BM México. 15 pp.

Grandin, T. 1997. Assessment of stress during handling and transport. J Anim Sci, 75: 249-257.

Grandin, T.; Oldfield, J.E. and Boyd, L.J. 1998. Review: Reducing handling stress improves both productivity and welfare. Profess Anim Scien, 14: 1-10.

Herrera, C.A. 2008. Análisis descriptivo de factores asociados a la presentación de contusiones y $\mathrm{pH}$ elevado en canales de bovinos de distinta procedencia geográfica. Memoria de título. Escuela de Medicina Veterinaria. Universidad Austral de Chile. Valdivia. 38 pp. Hood, D.E. and Tarrant, P.V. 1981. The problem of dark-cutting in beef. Martinus Niihoff Publishers BV. The Netherlands. 504 pp.

Knowles, T.G. 1999. A review of the road transport of cattle. Vet Rec, 144: 197-201.

Lenis, Y.; Zuluaga, A. and Tarazona, A. 2016. Adaptive responses to thermal stress in mammals. Arch Med Vet, 31: 121-135.

Martin, P. and Bateson, P. 2007. Measuring behaviour: An introductory guide. $3^{\text {rd }}$ ed. Cambridge University Press, Uk.

Miranda-de la Lama, G.C. 2013. Transporte y logística pre-sacrificio: principios y tendencias en bienestar animal y su relación con la calidad de la carne. Vet Méx, 44: 31-56.

Mota, D.; Velarde, A.; Huertas, S. y Cajiao, M.N. 2016. Bienestar animal. Una visión global en lberoamérica. $3^{\mathrm{a}}$ ed. Elsevier. México.

Muñoz, M. 2014. Bienestar animal: un reto en la producción pecuaria. Spei Domus, 10: 31-40.

Oddy, V.H.; Harper, G.S.; Greenwood, P.L. and McDonagh, M.B. 2001. Nutritional and developmental effects on the intrinsic properties of muscles as they relate to the eating quality of beef. Aust J Exp Agr, 41: 921. 
Paranhos da Costa, M.J.R. y Tarazona A. 2011 . Abordaje práctico sobre cómo mejorar el bienestar en los bovinos. RCCP, 24: 347.

Petryna, A. y Bavera, G.A. 2002. Etología. Cursos de producción bovina de carne, FAV UNRC. En: http://www.produccion-animal.com.ar/ etologia_y_bienestar/etologia_en_general/07-etologia.pdf

Quiroz, K.; Restrepo, D. y Barahona, R. 2016. Efecto Del tiempo de ayuno sobre el rendimento en canal y el pH em canales bovinas. Rev Lasallista Investig, 13: 80-87.

Romero, M. y Sanchéz, J. 2011 . Biomarcadores de estrés como indicadores de bienestar animal en ganado de carne. Biosalud, 10: 71-87.

Romero, M.; Uribe, L.; Sanchez, J. and Miranda de la Lama, G. 2013. Risk factors influencing bruising and high muscle $\mathrm{pH}$ in Colombian cattle carcasses due to transport and pre-slaughter operations. Meat Sci, 95: 256-263.

Sanmiguel, R. y Díaz, A. 2011 . Mecanismos fisiológicos de la termorregulación en animales de producción. RCCP, 4: 88-94.

Soria, L. and Corva, P. 2004. Factores genéticos y ambientales que determinan la terneza de la carne bovina. Arch Latinoam Prod Anim, 12: 73-88.
Tarazona, A. 2013. Efecto de diferentes arreglos silvopastoriles sobre el comportamiento de consumo y el bienestar animal y su relación con la disipación de la energía en sistemas de producción bovina. Tesis doctoral. Doctorado en Ciencias Animales. Universidad de Antioquia. Van de Water, G.; Verjans, F. and Geers, R. 2003. The effect of short distance transport under commercial condition on the physiology of slaughter calves; pH ad color profile of veals. Livest Prod Sci, 82: 171-179.

Warriss, P.D. 1992. Animal welfare. Handling animals before slaughter and the consequences for welfare and product quality. Meat Focus International. CAB International. Wallingford, UK. pp. 135-138.

Warriss, P.D.; Brown, S.N.; Knowles, T.G.; Kestin, S.C.; Edwars, J.E.; Dolan, S. K. and Phillips, A.J. 1995. Effects on cattle of transport by road for up to 15 hours. Vet Rec, 136: 319-323.

Warriss, P.D. 2000. Meat Science: An introductory text. CABI Publishing. New York. 310 pp. 